\title{
The Snake-in-the-Torus parameter-optimization algorithm: Initial evaluation
}

\author{
F. MICHAEL RABINOWITZ \\ Memorial University of Newfoundland, St. John's, Newfoundland, Canada
}

\begin{abstract}
A new quasi-annealing parameter-optimization algorithm, which can be implemented with parallel processors, is explained. The performance of this algorithm is compared to that of the Simplex algorithm on a variety of nonlinear functions with constraints. In general, the algorithm is more robust, but computationally more expensive, than the Simplex algorithm. General issues discussed include algorithm speed and robustness, applicability to both computer and mathematical models, and parallel efficiency.
\end{abstract}

Parameter estimation procedures can be used to find roots and either minimize or maximize a function in a specified domain. Some procedures are only applicable to mathematical functions with first and second derivatives, whereas other procedures require only that the function return a value. The latter procedures can also be used to estimate parameters in computer programs. In psychological research, parameter optimization is useful for obtaining maximum likelihood estimates, calculating least squares fits when exact mathematical solutions do not exist, and optimizing the fit of computer simulations to data.

Generally, the problem of finding roots is easier to solve than the problem of locating global maxima or minima. If a function is continuous and one can find parameter sets that return negative and positive values, then a root must lie somewhere between these parameter sets. The Bisection procedure takes advantage of this fact and is generally successful in locating roots. It is less useful, however, for locating maxima or minima (see Rabinowitz, Grant, \& Dingley, 1984, for additional discussion).

Locating maxima and minima are symmetrical problems. Therefore, only minimization problems are discussed here. A large number of optimization algorithms can usually solve such problems in $n$-space, where $n$ is the number of parameters, if the function is unimodal. Multimodal functions prove much more difficult. In the multimodal case, many algorithms exit at the first minimum found. For most problems, an exhaustive or grid search of the parameter space to locate a global minimum is impractical, because it would involve too large a number of function evaluations. Therefore, some form of limited search, involving random and/or systematic procedures, is used (see Press, Flannery, Teukolsky, \& Vetterling,

Preparation of this article was supported by Grant OGP0002017 from the Natural Sciences and Engineering Research Council of Canada. Special thanks are due Avery Earle, Malcolm Grant, and Mark Howe for their help and encouragement. Requests for reprints should be addressed to the author at the Department of Psychology, Memorial University of Newfoundland, St. John's, Newfoundland A1B 3X9, Canada.
1988 , for a discussion and description of many optimization procedures).

In order to illustrate why finding a minimum value of a function is often difficult, consider the Rosenbrock function,

$$
f(x, y)=100\left(y-x^{2}\right)^{2}+(1-x)^{2} .
$$

The negative of the fourth root of this function is illustrated in Figure 1 (range of $x,-5$ to 5; range of $y, 0$ to 10). In order to illustrate the infinite number of local minima, it was necessary to invert the function (making minima appear as maxima avoids masking these values) and to use the fourth root (which markedly restricts the range of function values). Note that there are two sharply defined minima for every value of $y$. The problem faced by an optimization procedure is to locate the point $(1,1)$, the global minimum, in a space where $x$ and $y$ are permitted to vary continuously. This is not easy to achieve, because when an algorithm finds a sharply defined local minimum, almost any change in parameter values will return a markedly increased function value. For example, the point $(10,100)$ returns a Rosenbrock function value of 81 , but the point $(4,2)$, which is much closer to the global minimum, returns a function value of 25,609 .

\section{The Simplex Algorithm}

One of the more widely used algorithms to solve minimization problems in $n$-space, where $n \geq 2$ parameters, is the Simplex (Nelder \& Mead, 1965). The algorithm is fairly robust in that it finds the minima for many continuous functions. It also is relatively efficient, because it does not require a large number of function evaluations, in comparison with the Monte Carlo-based algorithms described below. If one is interested in finding solutions for parameters in computer models, it also has the desirable property that it does not require derivatives in order to operate.

The Simplex algorithm is conceptually simple, elegant, and geometrically based. A simplex is established using $n+1$ points in $n$-space. The point generating the highest function value is replaced in the next iteration by reflecting it over the centroid (i.e., the point with coordinates 


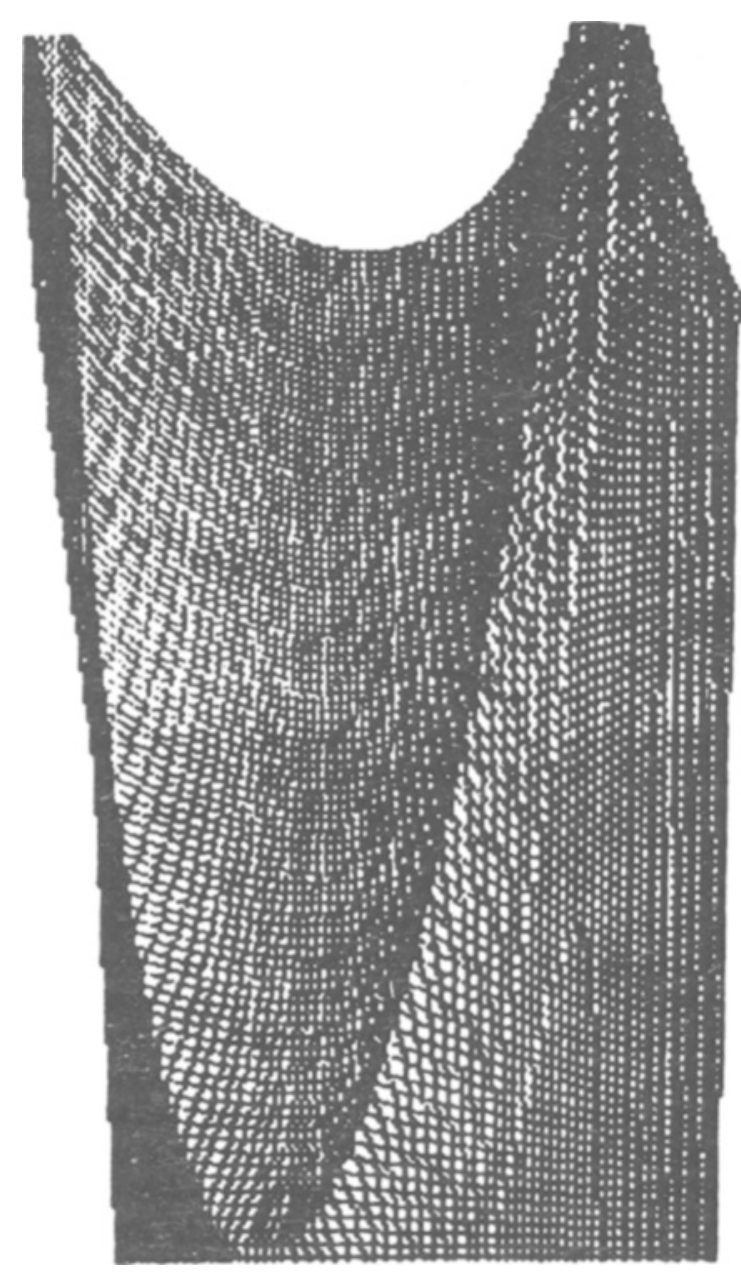

Figure 1. The negative of the fourth root of the Rosenbrock function.

equal to the mean values of the coordinates of each relevant point) determined by the remaining $n$ points. In 2 -space, this means that a line is drawn from the highest point across the middle of the line established by the two remaining points. A variety of simple rules are then followed to generate the replacement point.

\section{Simulated Annealing}

In 1983, Kirkpatrick, Gelatt, and Vecchi introduced and operationalized an optimization procedure that they termed simulated annealing. The idea came from statistical mechanics. It is based on the observation that solutions when cooled slowly will develop orderly stable molecular structures as solids. The idea can be applied to combinatorial optimization problems in the following way. First, a new point is randomly sampled. If it generates a new minimum value, it is always accepted. If not, it is accepted when a random number between 0 and 1 is less than a probability defined by a mathematical function, usually the Boltzmann equation. Early in the iterative process, the mathematical function generates values near 1 , and most points are accepted. By adjusting a parameter in the probability function, usually referred to as temperature, the probability function generates smaller values across successive iterations (cooling), and eventually, only points that produce better solutions are accepted. The procedure generates solutions for combinatorial problems-such as the problem of the traveling salesman-that are at present mathematically intractable. The success of the method appears to be a consequence of the exploration of a large number of combinations, as well as the small probability of being trapped by local minima until late in the iterative process.

Corana, Marchesi, Martini, and Ridella (1987) extended simulated annealing ideas to continuous functions. Note that, in combinatorial problems, there is a finite number of points that can be sampled. In comparison, there are an infinite number of possible points in continuous space. Corana et al. demonstrated that their procedure was robust, but it was very inefficient in a series of comparisons involving the Simplex algorithm (Nelder \& Mead, 1965), the Adaptive Random Search algorithm (Masri \& Bekey, 1980), and Simulated Annealing. In general, the Adaptive Random Search algorithm performed poorly on all their problems.

On Corana et al.'s (1987) series of tests involving variants of the Rosenbrock function, the Simulated Annealing algorithms solved all 17 problems, whereas the Simplex algorithm solved 15 of the 17 problems. However, the number of function evaluations varied from 161 to 1,869 using Simplex, but from 488,001 to $1,328,001$ using Simulated Annealing. On most Rosenbrock problems, the Simulated Annealing algorithm required from 500 to 1,000 times as many function calls as the Simplex procedure did.

Corana et al. (1987) constructed a parabolic minimization function for a second series of tests. In general, none of the 26 variants used was solved by the Simplex algorithm, but all were solved by the Simulated Annealing algorithm, using from 656,000 to $1,665,000$ function evaluations. The reader might note that the Snake-inthe-Torus algorithm described below also solved all variants of the parabolic minimization function, using a maximum of 15,000 function evaluations in each of four parallel processors.

\section{The Snake-in-the-Torus Algorithm}

Two concerns motivated the development of the Snakein-the-Torus algorithm. First, simulated annealing procedures tend to converge slowly, because many blind alleys are explored due to the stochastic nature of the acceptance process. Parameter sets that yield poorer function evaluations are particularly likely to be accepted early in the iterative process. The exploration of blind alleys, however, usually ensures an adequate search of the parameter space (i.e., not being trapped by local minima). As a consequence of this search, simulated annealing is a robust procedure. Therefore, it was desirable to develop a quasi-annealing procedure, capable of adequately exploring the parameter space, but less likely to explore blind alleys. Second, the simulated annealing procedures cur- 
rently implemented are serial (i.e., each iteration is dependent on and must sequentially follow the one that precedes it). Thus, parallel computer architectures offer little to speed up these computationally intensive algorithms. A parallel quasi-annealing algorithm offers the possibility that large multidimensional problems might be solvable in real time on parallel computers.

The Snake-in-the-Torus is a quasi-annealing algorithm. It consists of two basic procedures. The first is used in both $n$-space and 1-space. New points, neither too close to nor distant from the earlier point (i.e., falling in an $n$-dimensional torus with the earlier point as center), are selected by randomly generating values that are added to or subtracted from the coordinates of the earlier point. Any point generating a better fit than the previous bestfitting point becomes the center of a new $n$-dimensional torus. However, if a point does not generate a better fit, it is still used as the center for the next randomly selected point unless any $k$ (empirically values of $k$ between 6 and 12 are satisfactory; $k=10$ was used) consecutive points all fail to generate a better fit. In the latter case, sampling again begins with the best point as the center of the torus. In this way, the procedure fairly efficiently "snakes" around the predefined parameter space. The second procedure controls the size of the parameter space and alternates between $n$-dimensional and 1-dimensional application of the first procedure. A particularly important feature of the second procedure is to move the center of the torus off of a local minimum before the $n$-dimensional procedure is used. This is accomplished by selecting one variable and bumping it by either adding or subtracting $1 / 16$ (empirically values of $1 / 8$ and $1 / 32$ were less satisfactory) of the current range of the variable.

The algorithm is well suited to parallel implementation. The procedure currently used is to start each of $n$ processors at the same point. The best value returned is then used by all processors as the starting point for the next iteration. Torn and Zilinskas (1989) refer to this as Monte Carlo parallelism and note that it is easily implemented. It is also an efficient form of parallelism, in that each processor does the same amount of work. As is apparent below, parallel implementation of the algorithm markedly reduces the number of function calls per CPU for most problems. There is also some improvement in robustness.

The Snake-in-the-Torus algorithm is more flexible than most other optimization procedures. The success of the algorithm does not depend on the availability of derivatives, nor does it require that the function be continuous. If the algorithm is run several times on the same data set, then multiple solutions, if they exist, will often be revealed. (See Table 9 for an example.) This permits the user to select the solution that is most convenient to implement. Furthermore, the algorithm can handle integer as well as continuous parameter values. The latter property means that the algorithm can be used to optimize integer variables in psychological models. This is an important feature, because many psychological processes, such as depth of search of a game-board position, can only be meaningfully represented by integers.

\section{Some Test Problems}

In order to demonstrate that the Snake-in-the-Torus algorithm is potentially useful to psychologists, a series of test problems was chosen in which the Simplex algorithm (which is frequently used by mathematical psychologists) would be expected to perform well. All test functions were continuous and had first and second derivatives. All problems involved at least one equality or one inequality constraint. The first 30 problems in the Hock and Schittkowski (1981) collection of test examples for nonlinear programming codes were used. The Simplex algorithm solved 22 of the 30 problems.

Since the Snake-in-the-Torus algorithm is stochastic and representative median values were sought, 9 runs were used for all problems except Problem 25. This problem involved a complex summation (see Table 7) and was computationally expensive. Therefore, only one run was used. Across the 30 problems, the 1-cpu (4-cpu) algorithm solved 17 (25) problems without error (i.e., all 9 runs were successful with each problem). Furthermore, with the exception of the 1-cpu algorithm on Problem 15, the median value of the 9 runs provided a solution to all 30 problems for both the 1- and the 4-cpu versions of the algorithm. (The 1-cpu and 4-cpu versions of the algorithm failed on 31 and 11 of 262 runs, respectively.) The 1-cpu version of the Snake-in-the-Torus algorithm solved Problem 15 (see Table 3) on only 2 of the 9 runs, whereas the 4-cpu version of the algorithm solved this problem on 5 of 9 runs.

Four of the 30 problems, Problems 8, 9, 26, and 29, had more than one global minimum. With the exception of Problem 26 with the 1-cpu version, more than one minimum was discovered for each of these problems across the 9 runs.

The eight problems not solved by the Simplex algorithm, along with the problem solved on only 2 of 9 runs by the 1-cpu version of the Snake-in-the-Torus algorithm appear in Tables 1-9. Median values are reported for both versions of the Snake-in-the-Torus algorithm. Note that the Simplex algorithm did not return a value for Problem 25 (see Table 7), because it produced parameter values that could not be used to generate a function value.

\section{Discussion}

Tables 1-9 are representative of the general nature of the findings. The Simplex algorithm is less robust (i.e., it solves fewer problems) but more efficient (i.e., it uses fewer function calls) than the Snake-in-the-Torus algorithm. If the Simplex is providing correct solutions to a class of optimization problems, one would be ill advised to replace it. At best, an algorithm like the Snake-inthe-Torus could be used either initially or as an occasional check to establish that the Simplex is returning global minima. 
Table 1

Problem 3

Function: $\quad y+10^{-5}(y-x)^{2}$

Constraints: $0 \leq y$

\begin{tabular}{lrrrr}
\hline & $n$ & $f(x, y)$ & \multicolumn{1}{c}{$x$} & \multicolumn{1}{c}{$y$} \\
\hline Starting values & & 1.001 & 10.0 & 1.0 \\
Lower bounds & & 0.001 & -10.0 & 0.0 \\
Upper bounds & & 10.000 & 10.0 & 10.0 \\
True minimum & & 0.000 & 0.0 & 0.0 \\
Algorithm & & & & \\
Simplex & 177 & 0.001 & -10.000 & 0.000 \\
Torus 1 & 240 & 0.004 & 0.004 & 0.004 \\
Torus 4 & 200 & 0.002 & -0.003 & 0.002 \\
\hline
\end{tabular}

Table 3

Problem 15

Function: $\quad 100\left(y-x^{2}\right)^{2}+(1-x)^{2}$

Constraints: $0 \leq x y-1, \quad 0 \leq x+x y, \quad .5 \geq x$

\begin{tabular}{lrrrr}
\hline & $n$ & $f(x, y)$ & $x$ & \multicolumn{1}{c}{$y$} \\
\hline Starting values & & 909.00 & -2.0 & 1.0 \\
Lower bounds & & 1210121.00 & -10.0 & -10.0 \\
Upper bounds & & 9506.50 & 0.5 & 10.0 \\
True minimum & & 306.50 & 0.5 & 2.0 \\
Algorithm & & & & \\
$\quad$ Simplex & 571 & 306.500 & 0.500 & 2.000 \\
Torus 1* & 17200 & 360.381 & -0.792 & -1.263 \\
Torus 4* & 6960 & 306.523 & 0.500 & 2.000 \\
\hline
\end{tabular}

* The Tonus 1 (Torus 4) algorithm solved Problem 15 on 2 (5) of 9 runs.

Table 5

Problem 20

Function: $\quad 100\left(y-x^{2}\right)^{2}+(1-x)^{2}$

Constraints: $x+y^{2} \geq 0, \quad x^{2}+y \geq 0$,

\begin{tabular}{lrrrr}
\multicolumn{4}{c}{$x^{2}+y^{2}-1 \geq 0, \quad-5 \leq x \leq .5$} \\
\hline Starting values & $n$ & $f(x, y)$ & \multicolumn{1}{c}{$x$} & \multicolumn{1}{c}{$y$} \\
Lower bounds & & 58.500 & -0.5 & 1.000 \\
Upper bounds & & 9506.500 & -0.5 & -10.000 \\
True minimum & & 38.199 & 0.5 & 10.000 \\
Algorithm & & & 0.50 & 0.866 \\
Simplex & 257 & 58.500 & -0.5 & 1.000 \\
Torus 1 & 16720 & 38.200 & 0.5 & 0.866 \\
Torus 4 & 8360 & 38.200 & 0.5 & 0.866 \\
\hline
\end{tabular}

Table 7

Problem 25

Function: $\quad \sum_{1}^{99}\left[f_{i}(x, y, z)\right]^{2}$

$$
\begin{aligned}
& f_{i}(x, y, z)=-.01 i+e^{\left\lfloor-(1 / x)\left(u_{i}-y\right)^{z}\right\rfloor} \\
& u_{i}=25+(-50 \ln (.01 i))^{2 / 3} \\
& i=1, \ldots, 99
\end{aligned}
$$

\begin{tabular}{|c|c|c|c|c|c|}
\hline & $n$ & $f(x, y)$ & $x$ & $y$ & $z$ \\
\hline Starting values & & 32.835 & 100.0 & 12.500 & 3.0 \\
\hline Lower bounds & & 32.831 & 0.1 & 0.000 & 0.0 \\
\hline Upper bounds & & 19.972 & 100.0 & 26.500 & 5.0 \\
\hline True minimum & & 0.000 & 50.0 & 25.000 & 1.5 \\
\hline
\end{tabular}

Constraints: $0.1 \leq x \leq 100 \quad 0 \leq y \leq 25.6 \quad 0 \leq z \leq 5$

\section{Algorithm}

Simplex

Torus 1

NO VALUE RETURNED

Torus 4

\begin{tabular}{rrrrr}
3600 & 0.000 & 54.277 & 24.859 & 1.525 \\
960 & 0.001 & 63.608 & 24.572 & 1.572 \\
\hline
\end{tabular}

Table 2

Problem 13

\begin{tabular}{lcrcr}
\hline Function: & $\begin{array}{l}(x-2)^{2}+y^{2} \\
\text { Constraints: }\end{array}$ & $(1-x)^{3}-y \geq 0$, & $0 \leq x$, & $0 \leq y$ \\
& $n$ & $f(x, y)$ & $x$ & $y$ \\
\hline Starting values & & 3.970 & 0.1 & 0.1 \\
Lower bounds & & 4.000 & 0.0 & 0.0 \\
Upper bounds & & 185.000 & 10.0 & 11.0 \\
True minimum & & 1.000 & 1.0 & 0.0 \\
Algorithm & & & & \\
Simplex & 131 & 2.959 & 0.311 & 0.327 \\
Torus 1 & 9040 & 1.001 & 1.000 & 0.000 \\
Torus 4 & 6280 & 1.000 & 1.000 & 0.000 \\
\hline
\end{tabular}

Table 4

Problem 18

Function: $\quad .01 x^{2}+y^{2}$

Constraints: $\quad x y-25 \geq 0, \quad x^{2}+y^{2}-25 \geq 0$.

\begin{tabular}{lcccr}
\multicolumn{4}{c}{$2 \leq x \leq 50}$, & $0 \leq y \leq 50$ \\
& $n$ & $f(x, y)$ & $x$ & $y$ \\
\hline Starting values & & 4.04 & 2.000 & 2.000 \\
Lower bounds & & 0.04 & 2.000 & 0.000 \\
Upper bounds & & 2525.00 & 50.000 & 50.000 \\
True minimum & & 5.0 & 15.811 & 1.581 \\
Algorithm & & & & \\
Simplex & 142 & 7.172 & 10.075 & 2.481 \\
Torus 1 & 12320 & 5.000 & 15.850 & 1.577 \\
Torus 4 & 4600 & 5.000 & 15.819 & 1.580 \\
\hline
\end{tabular}

Table 6

Problem 24

Function: $\quad y^{3}\left[(x-3)^{2}-9\right] / 46.77$

Constraints: $\quad x / 1.73-y \geq 0, \quad x+1.73 y \geq 0$,

\begin{tabular}{lrrrr}
\multicolumn{4}{c}{$-x-1.73 y+6 \geq 0, \quad x \geq 0, \quad y \geq 0$} \\
\hline Starting values & $n$ & $f(x, y)$ & \multicolumn{1}{c}{$x$} & \multicolumn{1}{c}{$y$} \\
Lower bounds & & -0.013 & 1.0 & 0.500 \\
Upper bounds & & 0.000 & 0.0 & 0.000 \\
True minimum & & 855.334 & 10.0 & 10.000 \\
Algorithm & & -1.000 & 3.0 & 1.732 \\
Simplex & 147 & -0.748 & 3.269 & \\
Torus 1 & 4320 & -0.999 & 3.000 & 1.577 \\
Torus 4 & 1520 & -0.998 & 3.000 & 1.732 \\
\hline
\end{tabular}

Table 8

Problem 27

Function: $\quad\left(y-x^{2}\right)^{2}+.01(x-1)^{2}$

Constraint: $\quad x+z^{2}+1=0$

\begin{tabular}{lrrrrr}
\hline & $n$ & $f(x, y)$ & $x$ & $y$ & \multicolumn{1}{c}{$z$} \\
\hline Starting values & & 4.01 & 2.0 & 2.0 & 2.0 \\
Lower bounds & & 12101.21 & -10.0 & -10.0 & -10.0 \\
Upper bounds & & 8100.81 & 10.0 & 10.0 & 10.0 \\
True minimum & & 0.04 & -1.0 & 1.0 & 0.0 \\
Algorithm & & & & & \\
Simplex & 114 & 55.710 & -0.5 & 0.249 & -0.5 \\
Torus 1 & 4320 & .040 & -1.0 & 0.999 & 0.0 \\
Torus 4 & 2220 & .040 & -1.0 & 0.999 & 0.0 \\
\hline
\end{tabular}


Table 9

Problem 29

\begin{tabular}{|c|c|c|c|c|c|}
\hline \multirow[t]{2}{*}{$\begin{array}{l}\text { Function: } \\
\text { Constraint: }\end{array}$} & \multicolumn{5}{|c|}{$\begin{array}{l}-x y z \\
-x^{2}-2 y^{2}-4 z^{2}+48 \geq 0\end{array}$} \\
\hline & $n$ & $f(x, y)$ & $x$ & $y$ & $z$ \\
\hline \multicolumn{2}{|c|}{$\begin{array}{l}\text { Starting values } \\
\text { Lower bounds } \\
\text { Upper bounds } \\
\text { True minimum* }\end{array}$} & $\begin{array}{r}-1.000 \\
1000.000 \\
-1000.000 \\
-22.627\end{array}$ & $\begin{array}{r}1.0 \\
-10.0 \\
10.0\end{array}$ & $\begin{array}{r}1.0 \\
-10.0 \\
10.0\end{array}$ & $\begin{array}{r}1.0 \\
-10.0 \\
10.0\end{array}$ \\
\hline \multicolumn{6}{|l|}{ Algorithm } \\
\hline $\begin{array}{l}\text { Simplex } \\
\text { Torus } 1 \\
\text { Torus } 4\end{array}$ & $\begin{array}{r}191 \\
27000 \\
11760\end{array}$ & $\begin{array}{l}-18.102 \\
-22.627 \\
-22.627\end{array}$ & $\begin{array}{l}2.530 \\
3.998 \\
3.998\end{array}$ & $\begin{array}{r}2.829 \\
-2.828 \\
-2.829\end{array}$ & $\begin{array}{l}-2.530 \\
-2.001 \\
-2.000\end{array}$ \\
\hline
\end{tabular}

*This problem has 4 solutions $(a, b, c),(a,-b,-c),(-a, b,-c)$, and $(-a,-b, c)$ where $a=4, b=2.828$, and $c=2$. The Torus 1 (Torus 4) algorithm yielded all four (three) of these solutions in nine runs.

Comparison of the 1- and 4-cpu versions of the Snakein-the-Torus algorithm reveals that the 4-cpu version is somewhat more robust and requires fewer function calls per processor. Torn and Zilinskas (1989) discuss several metrics to evaluate parallelism. Speedup (time to solve for 1 processor / time to solve for $m$ processors) and efficiency (speedup $/ m$ ) are adequate metrics for present purposes. Inspection of Tables 1-9 reveals that the speedup (efficiency) ranges from 1.2 to $3.75(.30$ to .94$)$ with a mean of 2.29 (.57). It remains an empirical question whether or not these promising gains with parallelism are maintained when the number of parameters is greater than 3 and when the number of parallel processors is greater than 4 .
The Snake-in-the-Torus algorithm is relatively robust and efficient compared to other Monte Carlo algorithms. It would seem to be a procedure of choice when multiplesolutions are of interest, when functions are not continuous, and when parameters represent integers, rather than continuous variables. It should also be considered when more efficient algorithms do not generate satisfactory solutions, when it is necessary to establish boundary conditions for each parameter so that the function of interest is computable, and when it is desirable to validate a more efficient procedure.

\section{REFERENCES}

Corana, A., Marchesi, M., Martini, C., \& Ridella, S. (1987). Minimizing multimodal function of continuous variables with the "Simulated Annealing" algorithm. ACM Transactions in Mathematical Software, 13, 262-280.

Hock, W., \& SchitTkowskI, K. (1981). Test examples for nonlinear programming codes. Lecture Notes in Economics \& Mathematical Systems, 187, 1-177.

Kirkpatrick, S., Gelatt, C. D., \& Vecchi, M. P. (1983). Optimization by simulated annealing. Science, 220, 671-679.

MASRI, S. F., BekeY, G. A. (1980). A global optimization algorithm using adaptive random search. Applied Mathematics \& Computation, 7, 353-375

NELDER, J. A., \& MEAD, R. (1965). A simplex method for function minimization. Computer Journal, 7, 308-313.

Press, W. H., Flannery, B. P., Teukolsky, S. A., VetterLING, W. T. (1988). Numerical recipes in C: The art of scientific computing. New York: Cambridge.

Rabinowitz, F. M., Grant, M. J., \& Dingley, H. L. (1984). FIT: An iterative parameter-estimation function in LISP. Behavior Research Methods, Instruments, \& Computers, 16, 307-314.

TORN, A., ZILINSKAS, A. (1989). Global optimization. Lecture Notes in Computer Science, 350, 1-255. 